\title{
Finite electric displacement simulations of polar ionic solid-electrolyte interfaces: Application to $\mathrm{NaCl}(111) / a q u e o u s ~ \mathrm{NaCl}$ solution ${ }^{\circledR P}$
}

Cite as: J. Chem. Phys. 150, 041716 (2019); https://doi.org/10.1063/1.5054843

Submitted: 04 September 2018 . Accepted: 05 November 2018 . Published Online: 14 December 2018

Thomas Sayer (D), Michiel Sprik (D), and Chao Zhang

\section{COLLECTIONS}

EP

This paper was selected as an Editor's Pick
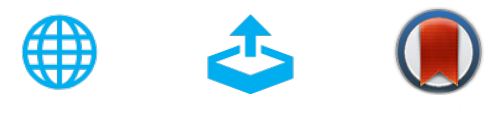

\section{ARTICLES YOU MAY BE INTERESTED IN}

Understanding the electrochemical double layer at the hematite/water interface: A first principles molecular dynamics study

The Journal of Chemical Physics 150, 041707 (2019); https://doi.org/10.1063/1.5047930

Grand-canonical approach to density functional theory of electrocatalytic systems: Thermodynamics of solid-liquid interfaces at constant ion and electrode potentials The Journal of Chemical Physics 150, 041706 (2019); https://doi.org/10.1063/1.5047829

A comparison of surface hopping approaches for capturing metal-molecule electron transfer: A broadened classical master equation versus independent electron surface hopping

The Journal of Chemical Physics 150, 041711 (2019); https://doi.org/10.1063/1.5050235

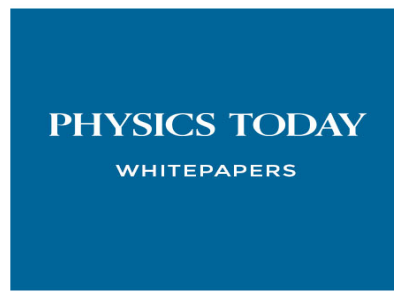

ADVANCED LIGHT CURE ADHESIVES

Take a closer look at what these environmentally friendly adhesive systems can do
READ NOW

PRESENTED BY 8 MASTERBOND: 


\title{
Finite electric displacement simulations of polar ionic solid-electrolyte interfaces: Application to $\mathrm{NaCl}\left(\begin{array}{lll}1 & 1 & 1\end{array}\right)$ /aqueous $\mathrm{NaCl}$ solution ${ }^{\circ}$
}

\author{
Cite as: J. Chem. Phys. 150, 041716 (2019); doi: 10.1063/1.5054843 \\ Submitted: 4 September 2018 • Accepted: 5 November 2018 • \\ Published Online: 14 December 2018
}

Thomas Sayer, ${ }^{1, a)}$ (D) Michiel Sprik, ' (D) and Chao Zhang ${ }^{2}$

AFFILIATIONS

'Department of Chemistry, University of Cambridge, Cambridge CB2 1EW, United Kingdom

${ }^{2}$ Department of Chemistry-Ångström Laboratory, Uppsala University, Lägerhyddsvägen 1, P.O. Box 538, 75121 Uppsala, Sweden

a) Electronic mail: tes36@cam.ac.uk

\begin{abstract}
Tasker type III polar terminations of ionic crystals carry a net surface charge as well as a dipole moment and are fundamentally unstable. In contact with electrolytes, such polar surfaces can be stabilized by adsorption of counterions from the solution to form electric double layers. In a previous work [T. Sayer et al., J. Chem. Phys 147, 104702 (2017)], we reported on a classical force field based molecular dynamics study of a prototype model system, namely, a $\mathrm{NaCl}(111)$ slab interfaced with an aqueous $\mathrm{NaCl}$ solution on both sides. A serious hurdle in the simulation is that the finite width of the slab admits an electric field in the solid perturbing the theoretical charge balance at the interface of semi-infinite systems [half the surface charge density for $\mathrm{NaCl}(111)]$. It was demonstrated that the application of a finite macroscopic field $E$ canceling the internal electric field can recover the correct charge compensation at the interface. In the present work, we expand this method by applying a conjugate electric displacement field $D$. The benefits of using $D$ instead of $E$ as the control variable are two fold: it does not only speed up the convergence of the polarization in the simulation but also leads to a succinct expression for the biasing displacement field involving only structural parameters which are known in advance. This makes it feasible to study the charge compensating phenomenon of this prototype system with density functional theory based molecular dynamics, as shown in this work.
\end{abstract}

Published under license by AIP Publishing. https://doi.org/10.1063/1.5054843

\section{INTRODUCTION}

Termination of ionic crystals can leave the solid with a surface carrying a net charge. While generally less stable when compared to (low index) uncharged surfaces, a net surface charge can still be accommodated, provided that the termination does not also create a surface dipole moment. This is the non-polar type II termination in the classification of Tasker. ${ }^{1}$ The familiar example is the (111) surface of $\mathrm{CaF}_{2}$ (fluorite). By contrast, the (111) termination of $\mathrm{NaCl}$ (rocksalt) is fundamentally polar and unstable. This is the type III termination in the classification of Tasker. ${ }^{7}$ Other examples of type III rocksalt surfaces are the (111) surfaces of $\mathrm{MgO}$ and $\mathrm{NiO}$. The (0001) surfaces of the corundum $\left(\mathrm{Al}_{2} \mathrm{O}_{3}, \mathrm{Fe}_{2} \mathrm{O}_{3}\right)$ and wurtzite $(\mathrm{ZnO})$ structure are also type III polar surfaces. For an extensive review of polar surfaces, we refer the reader to the 2008 review by Goniakowski et al. ${ }^{2}$

Type III polar surfaces can be stabilized by a surface reconstruction which eliminates the dipole moment. The reconstruction is necessarily non-stoichiometric as it must change the net surface charge. ${ }^{2}$ For polar interfaces with vacuum, the non-stoichiometric construction is often observed to occur by removal or addition of ions. Polar solid-solid interfaces also undergo an electronic reconstruction. For polar surfaces in contact with an electrolyte, the compensating charge can be provided by an exchange of ions with the electrolyte. In a previous publication, we have investigated such an ionic solid/electrolyte interface, $\mathrm{NaCl}(111) / \mathrm{NaCl}(\mathrm{aq})$, using classical force field based molecular dynamics (FFMD) simulation. ${ }^{3}$ 
This paper will be referred to as Paper I. ${ }^{3}$ In the present contribution, we return to the model system of Paper $\mathrm{I}^{3}$ now also applying density functional theory based molecular dynamics (DFTMD).

Atomistic modeling of type III interfaces is a major challenge. The key reason is the slab geometry used in modeling. Slabs of finite width can sustain the electric field created by the polarization and frustrate the reconstruction observed in semi-infinite systems. For physical nanosystems (thin films, nanoparticles), this behavior is real and of great technical as well as practical interest. ${ }^{4}$ In simulation studies aiming to understand the fate of polar surfaces of semi-infinite crystals, this effect must, however, be regarded as a finite size error. For typical model system dimensions used in atomistic simulation, this finite size effect can be rather serious.

A finite width slab would be a better representation of a semi-infinite system if it were possible to cancel out the internal electric field. This suggests application of an appropriate bias perpendicular to the surface. Finite external electric fields are relatively easy to implement for slabs in vacuum under open boundary conditions. The challenge is to apply a field to a polar crystalline slab immersed in an electrolyte maintaining full periodic boundary conditions in parallel as well as normal directions and without inserting false vacuum spacers. This was achieved in Paper $\mathrm{I}^{3}$ using the finite electric field methods developed by Vanderbilt and colleagues for treating periodic supercells of ferroelectric solids and multilayer systems. ${ }^{5-7}$

This compensating field method was already introduced in Ref. 8. There, it was used to study the electrostatics of the compact electric double layer (EDL) formed at the interface between a high concentration aqueous electrolyte $(\mathrm{NaCl})$ and a hard wall with a fixed surface charge. The system was made 3D periodic by introducing a second wall of opposite charge separated from the first wall by a vacuum space (a.k.a. the insulator). These walls are a simple model of a polarized insulating slab. Again, because of the finite width, the slab admits an electric field. The result is an uncompensated EDL with a net finite charge as can be shown by a simple application of the Maxwell interface theorem. ${ }^{8}$ We note that this finite size error must be distinguished from the interaction of a polarized slab with its images under periodic boundary conditions. Polarization of a finite width isolated slab (open boundary conditions) also induces internal electric fields leading equally to an EDL with a net charge.

The electrostatics of the model systems in Paper $\mathrm{I}^{3}$ as determined by FFMD were analyzed in detail by comparison to analytic results for the continuum model shown in Fig. 1. The system is periodic with the solid in the center. The zones of the electrolyte on the left- and right-hand side are part of the same layer of the electrolyte intersected by the boundaries of the supercell. The key simplification of the model in Fig. 1 is that the electrolyte is treated as a continuum with infinite dielectric constant separated from the surface of the solid slab by a layer with a finite dielectric constant $\epsilon_{\mathrm{H}}$. This polarizable continuum represents the compact Stern layer in a high concentration solution (no diffuse layer). $\sigma$ is the ionic surface charge density of the Helmholtz plane. It is the response of the electrolyte screening the surface charge distribution $\sigma_{0}$ of the ionic solid. In Fig. 1, the atomic point charges of the (111) plane have been smeared out into a homogeneous surface charge distribution $\pm \sigma_{0}$. The solid consists of $n+1$ of these alternating charge planes with $n$ as an odd integer. There are three geometric parameters in the model, the width $l_{\mathrm{H}}$ of the Stern layer, the spacing $R$ between the charge planes, and the repeat length $L$ of the periodic boundary conditions normal to the surface. The width of the solid slab equals $n R$. This leaves a width of $L-2 l_{\mathrm{H}}-n R$ for the zone of the electrolyte.

With $\sigma_{0}$ fixed and the potential across the solid slab tuned to zero by an appropriate biasing potential (see Sec. III), the central variable in the continuum model is the electrolyte surface charge $\sigma$. The model is a piecewise homogeneous system, and $\sigma$ can be determined by application of the Maxwell interface theorem. The solution, derived in Paper $\mathrm{I}^{3}$ and recapitulated in Sec. III, is

$$
\sigma_{\mathrm{CNC}}=\frac{n+1}{2 n} \sigma_{0}
$$

where CNC stands for compensating net charge. The CNC nomenclature was chosen as a generalization of the Zero Net Charge (ZNC) condition restoring the charge balance in the

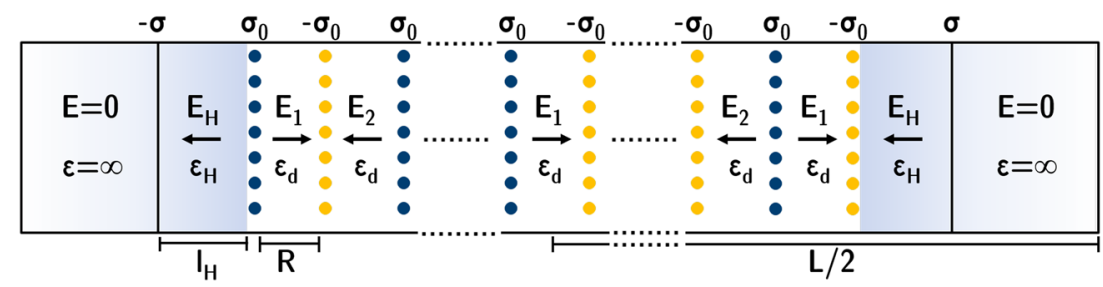

FIG. 1. Stern model of the ICS from Paper I. ${ }^{3}$ The (absolute) surface charge density of the polar surface's ions is $\sigma_{0}$, and the compensating charge induced in the electrolyte solution is $\sigma$. The solid slab is separated from the electrolyte on both sides by Helmholtz layers. The dielectric constants of the Helmholtz layers and polar solid are $\epsilon_{H}$ and $\epsilon_{\mathrm{d}}$, respectively. The periodic box size is $L$, the width of the Helmholtz layer is $I_{\mathrm{H}}$, and the thickness of a layer in the polar solid is $R$. The arrows indicate the convention for the sign of the uniform electric fields in the Helmholtz layers and crystal segments. We additionally note that $E_{1}$ is in the middle of the slab for $(n+1) / 2$ an odd integer; otherwise we have $E_{2}$. This does not influence the result for the ICS but will matter for the ECS discussion of Sec. IV. Reprinted with permission from Sayer et al., J. Chem. Phys. 147, 104702 (2017). Copyright 2017 AIP Publishing LLC. 
double layers of Ref. 8. Indeed setting $n=1$ in Eq. (1) gives $\sigma_{\mathrm{CNC}}=\sigma_{0}$. For $n \gg 1$ (the thick polar slab limit), $\sigma_{\mathrm{CNC}}=\sigma_{0} / 2$. Equation (1) interpolates between the neutral double layer system studied in Ref. 8 and that of the compensating charge predicted by the Tasker rule for a semi-infinite (111) polar rocksalt surface. This is to be expected. However, less obvious is that the semi-infinite limit is reached gradually. The number of planes in the crossover region is predicted to be independent of any geometric or dielectric parameters. Equation (1) must be regarded as a residual finite width effect persisting even after the average internal electric field in the slab has been canceled by an external bias.

The FFMD results of Paper $\mathrm{I}^{3}$ on the $\mathrm{NaCl}(111) / \mathrm{NaCl}(\mathrm{aq})$ model system are in excellent agreement with the predictions of Eq. (1), confirming that it takes a system with a minimum of 15 ionic planes to reduce the compensating electrolyte charge to the half-charge Tasker limit. Unfortunately, such system sizes exceed what is feasible in DFTMD. However, in view of the apparently parameter free $n$ dependence of Eq. (1), it seemed acceptable to us to reduce $n$ to a minimum value of $n=3$, which according to Eq. (1) would give a compensation charge of $\sigma=2 \sigma_{0} / 3$. This is approximately midway between the $\sigma=\sigma_{0}$ double layer limit and the $\sigma=\sigma_{0} / 2$ semi-infinite limit. We know from Paper $\mathrm{I}^{3}$ that the FFMD system satisfies this relation. The question is whether the DFTMD simulation does as well. Anticipating our results, the DFTMD and FFMD seem to agree upon the value of the net compensating charge while admitting significant differences in the structure of the interface.

The present contribution goes beyond Paper $\mathrm{I}^{3}$ in a further more important aspect. This concerns the feasibility of applying the constant field method in DFTMD simulation. The constant field that is imposed in Paper $\mathrm{I}^{3}$ is the macroscopic Maxwell field $\bar{E}$. $\bar{E}$ is a control parameter in the constant field Hamiltonian (see Sec. II A), and what is needed is the particular field $\bar{E}$ canceling the internal electric field across the solid slab. This field was referred to in Paper $\mathrm{I}^{3}$ as $\bar{E}=\mathrm{E}_{\mathrm{CNC}}$. $E_{\mathrm{CNC}}$ was empirically determined by searching for the $\bar{E}$ which eliminates the potential drop over the polar crystal slab. This requires a series of finite $\bar{E}$ calculations, and so the resulting computational overhead makes it prohibitive for DFTMD simulations.

Dielectric theory defines a second field conjugate to the Maxwell field E. This is the dielectric displacement $D$ related to the Maxwell field $\mathrm{E}$ as

$$
\mathrm{D}=\mathrm{E}+4 \pi \mathrm{P}
$$

where $\mathrm{P}$ is the polarization (as in previous publications, Gaussian electric units are used). The quantities D, E, and P in Eq. (2) are a short hand notation for the component of the corresponding vector fields perpendicular to the slab (see Fig. 1). As an alternative to the constant E method, Stengel, Spaldin, and Vanderbilt (SSV) also developed a constant D method. ${ }^{5}$ As the SSV constant E and D methods were intended for application in DFT based electronic structure calculation, the methods were formulated in terms of extended Hamiltonians coupling the polarization to a uniform Maxwell field or dielectric displacement. 5, 6 The crucial step in this development was the representation of electronic polarization in terms of a Berry phase, as in the modern theory of polarization. ${ }^{9-11}$ The same DFT approach will be applied in the present work. However, the extension term can also be used in combination with an FFMD Ewald Hamiltonian.

It is shown in Ref. 8 that keeping $D$ fixed is a more efficient method for determining $E_{\mathrm{CNC}}$ as will be explained again below. The constant D scheme was not used in Paper I, ${ }^{3}$ and this will be taken up in the present paper. Another proposed advantage is that while $\mathrm{E}_{\mathrm{CNC}}$ depended on the capacitance of the (Helmholtz) double layer $\mathrm{C}_{\mathrm{H}}$-the determination of which is usually an objective of the simulation-the value of $\mathrm{D}_{\mathrm{CNC}}$ involved only structural parameters. In this work, we will derive the expression of $\mathrm{D}_{\mathrm{CNC}}$, validate it with FFMD simulations of $\mathrm{NaCl}(111) / \mathrm{NaCl}$ solution, and then apply it to DFTMD simulations of exactly the same system. The present contribution is predominantly devoted to establishing the methodology for treating periodic systems with spontaneous polarization and to testing its feasibility in the context of DFTMD simulations (theory and implementation). This is the subject of Secs. II A-V. The focus of property calculation is therefore on the capacitance and dielectric response (Sec. VI). A detailed structural analysis is deferred to follow-up publications.

\section{CONSTANT FIELD MOLECULAR DYNAMICS}

\section{A. Constant field Hamiltonians}

In this section, we briefly summarize the constant $E$ and constant D method in order to highlight some important technical issues. We first outline the FFMD implementation as used in Paper $\mathrm{I}^{3}$ and Ref. 8. In the present investigation, this method is again used for initialization of the DFT simulation and for comparison to the DFT results. The adaption of SSV constant methods for FFMD simulation of aqueous systems is relatively straightforward. ${ }^{12}$ The constant-E Hamiltonian is written as

$$
\mathrm{H}_{\mathrm{E}}=\sum_{i}^{\mathrm{N}} \frac{\mathbf{p}_{i}^{2}}{2 m_{i}}+\mathrm{V}_{\mathrm{PBC}}\left(\mathbf{r}^{\mathrm{N}}\right)-\frac{\Omega}{8 \pi} \mathbf{E}^{2}-\Omega \mathbf{E} \cdot \mathbf{P}
$$

and the constant-D Hamiltonian as

$$
\mathrm{H}_{\mathrm{D}}=\sum_{i}^{\mathrm{N}} \frac{\mathbf{p}_{i}^{2}}{2 m_{i}}+\mathrm{V}_{\mathrm{PBC}}\left(\mathbf{r}^{\mathrm{N}}\right)+\frac{\Omega}{8 \pi}(\mathbf{D}-4 \pi \mathbf{P})^{2},
$$

where $\mathbf{p}_{i}=m_{i} \mathbf{v}_{i}$ is the momentum of particle $i$ with the position vector $\mathbf{r}_{i}$ and velocity $\mathbf{v}_{i}=\dot{\mathbf{r}}_{i}$. The mass of particle $i$ is $m_{i}$. The potential $V_{\mathrm{PBC}}\left(\mathbf{r}^{\mathrm{N}}\right)$ is the potential energy of the $\mathrm{N}$ particle system with the electrostatic interactions evaluated using standard Ewald summation (no surface term). ${ }^{13,14} \Omega$ is the volume of the periodic supercell.

\section{B. Polarization in ionic systems}

Polarization in a periodic system is multivalued in nature. ${ }^{9-11}$ For example, whenever a unit of charge $e$ wraps 
across the supercell boundary in the $z$ direction, there is a jump in the polarization. This jump $\Delta_{q} P$, termed the "quantum of polarization," is a simple function of the supercell dimensions,

$$
\Delta_{q} \mathrm{P}=\frac{e \mathrm{~L}}{\Omega} .
$$

Equation (5) is valid for any orthorhombic supercell of ions and electrons. For our model system of a rigid polar crystal oriented perpendicular to the $z$ axis (Fig. 1), $\Delta P_{q}$ can be equated to

$$
\Delta_{q} \mathrm{P}=\frac{\sigma_{0}}{\mathrm{~N}_{0}}
$$

and $\mathrm{N}_{0}$ is the number of ions in a charged plane. Equation (6) is the more convenient expression to be used later in the calculations.

Polarization in periodic systems is defined modulo the quantum of polarization $\Delta_{q} P$. This uncertainty is reflected in the constant E Hamiltonian of Eq. (3) (also called the electrostatic enthalpy) which is now also multiple valued. Adopting the language of mathematics, the energy surface consists of a stack of branches, each branch defined by a multiple of $\Delta_{q} P$. By contrast, the constant $\mathbf{D}$ is free from this ambiguity in principle, provided that the $\Delta_{q} P$ for a given branch of polarization is deducted again from the displacement field. The branches collapse on a single energy surface given by Eq. (4). Indeed the quantity in the brackets of $H_{D}$ is the electrostatic field [see Eq. (2)]. $q \mathbf{E}$ is a force on a particle with charge $q$ and therefore uniquely defined. The value of a displacement field in a supercell is therefore also multivalued. ${ }^{6}$

E in Eq. (3) and D in Eq. (4) are parameters (control variables), and that is why we put bar over them as $\bar{E}$ and $\bar{D}$ in previous publications. ${ }^{3,12}$ The polarization $\mathbf{P}$ is a system observable fluctuating in time. For liquid water, $\mathbf{P}$ is simply the sum of the molecular dipole moments. ${ }^{12}$ For application to ionic solutions, all charge, bound (solvent) and mobile (ions), must be included in the polarization. ${ }^{8}$ This introduces the confusing complication of multiple values and branches described above. The contribution to polarization of the free ions now depends on the choice of supercell. ${ }^{6,8}$ During the time evolution, ambiguity is avoided by following an ion when it crosses a supercell boundary. The value of the polarization is therefore fixed by where the ions are in the supercell of choice at the start of the MD trajectory. Formally, this definition of polarization can be regarded as an integral of the (total) current and is referred to as itinerant polarization as was already introduced earlier in FFMD simulation of homogeneous ionic solutions. ${ }^{13,15,16}$

The dependence of the polarization on the choice of the unit cell is a necessary artefact. It can be regarded as a gauge. Experimentally observable quantities, such as the electrolyte response charge $\sigma$, should be invariant under a change of supercell. As discussed in Ref. 8, the geometry of alternating solid slabs and electrolytes leads to two fundamentally different options for a supercell. The first is a configuration with the slab in the middle and the cell boundaries partitioning the electrolyte in two half's, one to the left and one to the right of the central slab. This is the configuration depicted in Fig. 1 and was referred in Ref. 8 as the insulator centered supercell (ICS). The alternative is placing the electrolyte in the middle and the solid on the side giving an electrolyte centered supercell (ECS).

The crucial difference between the ICS and ECS geometries is that in the ECS, the ions are confined by the solid surfaces and cannot escape from the cell. The same supercell can be used to specify the polarization over the entire run eliminating any ambiguity. In the ICS, mobile ions can cross the cell boundaries and we will have to keep track of where they go in order to refer back to their initial position, which was the basis of the definition of polarization. Clearly, the MD results obtained in an ICS or ECS must be consistent. This consistency test was the main tool used to validate the FFMD SSV finite field supercell approach in Ref. 8. The freedom of switching between ECS and ICS when this is convenient will be exploited in the present calculation.

\section{CONTINUUM STERN MODEL}

To work out the relevant equations for the continuum approximation of Fig. 1, we employ a generalization of the derivation for the more simple EDL model of Ref. 8. In Sec. III A, we first derive the expression for the full polarization $P$ and electric field $\bar{E}$ for the insulator centered cell (ICS) geometry of Fig. 1. Then, applying the constitutive relation assumed in the continuum model, we obtain an equation for the compensating internal field $\mathrm{E}_{\mathrm{CNC}}$ of the polar crystal which will lead to Eq. (1). Everything up to this point was already derived in Paper I. ${ }^{3}$ We are then in a position to transform $E_{\mathrm{CNC}}$ to the corresponding dielectric displacement $\mathrm{D}_{\mathrm{CNC}}$ according to Eq. (2). Finally, we analyze the equation of state, expressing the capacitance of the Helmholtz layer, $\mathrm{C}_{\mathrm{H}}$ in terms of the polarization at $\mathrm{CNC}$.

This leads directly to Sec. IV, where we go on to consider what steps need to be taken to reconcile our model with the gauge uncertainty of Sec. II B as it manifests in a DFT calculation, that is to say the Berry phase polarization. We explain why the ECS is the natural choice for an MD simulation and explore how this affects the value of $\mathrm{D}_{\mathrm{CNC}}$.

\section{A. Polarization}

We begin by writing down the total surface charge density $\sigma_{\mathrm{H}}$ of the Helmholtz plane separating the electrolyte from the Stern layer. In other words, we consider the dielectric response to the electrostatic geometry given by Fig. 1. Applying the Maxwell interface theorem to the first (or last) plane of charge in Fig. 1 yields

$$
\sigma_{\mathrm{H}}=\sigma-\mathrm{P}_{\mathrm{H}} .
$$

In words, this is the (ionic) response of the electrolyte to the presence of the surface $\sigma$, plus the surface contribution from polarization of the bound charge (solvent) making up the dielectric material of the Stern layer $\mathrm{P}_{\mathrm{H}}$. Note that the sign of the polarization follows the convention for the electric field, which changes sign from one layer to the next, as indicated by the arrows in Fig. 1. 
Polarization of the Stern layer will also add to, or subtract from, the fixed charge $\pm \sigma_{0}$ on the outermost crystal planes. Similar to Eq. (7), the net charge density of the terminal crystal plane would therefore be the sum of the fixed charge density $\sigma_{0}$ and the charge density induced at the bounding surface of the Stern layer. Furthermore, we assume that the gaps between the crystal planes also behave as a dielectric material. The polarization between the crystal planes is therefore finite. Labeling the outer plane on the left (blue dots) by index $i=1$, we have for its net charge density

$$
\sigma_{1}=\sigma_{0}-\mathrm{P}_{\mathrm{H}}-\mathrm{P}_{1}
$$

where $P_{1}$ is the polarization between outer plane 1 and plane 2 , the next plane in from plane 1.

While the dielectric spacers consist of the same material everywhere, the polarization inside the crystal is not uniform. As a result, the polarization $\mathrm{P}_{2}$ in the layer between plane $i=2$ and the next plane in (plane $i=3$ ) can be different from $P_{1}$. The negative charge of the first subsurface plane from the left (yellow dots) is therefore

$$
\sigma_{2}=\sigma_{0}-\mathrm{P}_{1}-\mathrm{P}_{2} .
$$

As the fixed charge of the crystal planes alternates between $+\sigma_{0}$ and $-\sigma_{0}$, the Maxwell interface theorem for the series of planes $i=2, \ldots, n$ is satisfied by a charge of $\sigma_{2}$, switching sign from one plane to next, finally ending with $-\sigma_{1}$ for plane $i=n$ +1 on the right.

The net surface charge densities of Eqs. (7)-(9) are sufficient information to evaluate the polarization and Maxwell electric field of the continuum model of Fig. 1. The polarization is obtained from the total dipole moment,

$$
\mathrm{P}_{\text {cell }}^{\mathrm{ICS}}=\frac{1}{\mathrm{~L}}\left(\sigma_{\mathrm{H}}\left(2 l_{\mathrm{H}}+n \mathrm{R}\right)+\sum_{i=1}^{n+1} \sigma_{i} z_{i}\right),
$$

where $z_{i}$ is the location of the plane $i$ with surface charge density $\sigma_{i}$. We have added a superscript ICS to indicate that the polarization of Eq. (10) is specific for the insulator centered supercell of Fig. 1. The role of the subscript "cell" will become clear when we consider possible further contributions to the polarization from the electrostatic boundary conditions. Substitution of Eqs. (7)-(9) gives

$$
\mathrm{P}_{\text {cell }}^{\mathrm{ICS}}=\frac{1}{\mathrm{~L}}\left(\sigma_{\mathrm{H}}\left(2 l_{\mathrm{H}}+n \mathrm{R}\right)-\sigma_{1} n \mathrm{R}+\sigma_{2} \frac{n-1}{2} \mathrm{R}\right) .
$$

Recall that $n$ is an odd integer. Note also that the polarization includes the dipole moment arising from the fixed charge density.

The Maxwell field $\bar{E}$ is defined as $\Delta V=-L \bar{E}$ where $\Delta V$ is the potential across the cell. $\Delta \mathrm{V}$ is obtained by adding all the potentials over the piecewise uniform partitions of the cell. Taking into account the convention for the sign of the fields specified in Fig. 1, we find

$$
L \bar{E}=-2 l_{H} E_{H}+\left(\frac{n+1}{2}\right) R E_{1}-\left(\frac{n-1}{2}\right) R E_{2} .
$$

$\bar{E}$ can be finite leading to a finite potential over a periodic cell, which may at first seem to violate periodic boundary conditions. This is, however, a central feature of the SSV method avoiding open boundary conditions in condensed systems. ${ }^{6}$

The polarization of Eq. (11) can be related to the Maxwell field $\bar{E}$ by replacing the surface charge densities by the uniform fields in the subsystems obtained employing the Maxwell interface equation at their boundaries,

$$
\begin{aligned}
4 \pi \sigma_{\mathrm{H}} & =\mathrm{E}_{\mathrm{H}}, \\
4 \pi \sigma_{1} & =\mathrm{E}_{\mathrm{H}}+\mathrm{E}_{1}, \\
4 \pi \sigma_{2} & =\mathrm{E}_{2}+\mathrm{E}_{1},
\end{aligned}
$$

where we have used that the field in the electrolyte is strictly zero in our simple model. Equations (13)-(15) are equivalent to Eqs. (7)-(9) formulated in terms of electric fields instead of polarization. Inserting in Eq. (11) gives

$4 \pi \mathrm{P}_{\text {cell }}^{\mathrm{ICS}}=\frac{1}{\mathrm{~L}}\left(\mathrm{E}_{\mathrm{H}}\left(2 l_{\mathrm{H}}+n \mathrm{R}\right)-\left(\mathrm{E}_{\mathrm{H}}+\mathrm{E}_{1}\right) n \mathrm{R}+\left(\mathrm{E}_{1}+\mathrm{E}_{2}\right)\left(\frac{n-1}{2}\right) \mathrm{R}\right)$.

Comparing to Eq. (12), we conclude that

$$
4 \pi \mathrm{P}_{\text {cell }}^{\mathrm{ICS}}=-\overline{\mathrm{E}} .
$$

Equation (17) has the familiar form of the electric field generated by the polarization. At the first glance, this suggests that $\bar{E}$ is a polarization field. However, as defined by Eq. (12), $\bar{E}$ is the Maxwell field including a possible contribution from an applied field (commonly referred to as $E_{0}$ ). In Ref. 8, the resolution of this apparent inconsistency is that $\mathrm{P}_{\text {cell }}^{\mathrm{ICS}}$ is not the full polarization. What is missing is a surface term introduced by the supercell boundary. For the ICS, this boundary is located in the electrolyte. The polarization in the electrolyte is uniform and therefore the same as the polarization at the boundary with the Stern layer. This is the induction charge $-\sigma$, and hence we must write for the full polarization

$$
\mathrm{P}^{\mathrm{ICS}}=-\sigma+\mathrm{P}_{\text {cell }}^{\mathrm{ICS}}
$$

or in terms of the Maxwell field using Eq. (17),

$$
4 \pi \mathrm{P}^{\mathrm{ICS}}=-4 \pi \sigma-\overline{\mathrm{E}} .
$$

The need for surface terms related to periodic supercell boundaries is an important theme in solid state physics. ${ }^{17}$ In the physical chemistry literature on the simulation of polar liquids, this surface is often viewed as the polarization charge induced on the tinfoil boundaries at infinity. ${ }^{12,14}$

The derivation to this point is a recapitulation of the argument in Paper I. ${ }^{3}$ We now go beyond Paper $\mathrm{I}^{3}$ by making the link to the dielectric displacement $\mathrm{D}$. As was pointed out, all charge in the system, including the fixed charge $\sigma_{0}$, is accounted for in the polarization Eq. (11). There is no external charge. The value of $\mathrm{D}$ can therefore simply be obtained by adding the polarization to the field according to Eq. (2), 


$$
\mathrm{D}^{\mathrm{ICS}}=\overline{\mathrm{E}}+4 \pi \mathrm{P}^{\mathrm{ICS}} \text {. }
$$

We have attached a superscript ICS anticipating that the dielectric displacement, like the polarization, depends on the choice of supercell. Substituting Eq. (18), we find

$$
\mathrm{D}^{\mathrm{ICS}}=-4 \pi \sigma .
$$

Compared to the complexity of the expression for the Maxwell field [Eq. (12)], the equation for D is surprisingly simple, which is another reason for preferring the constant $D$ over the constant E method. Note, however, that the surface charge density determining the displacement field in Eq. (21) is the induced charge $\sigma$, not the fixed charge $\sigma_{0}$, which would have been the expected value if the fixed charge had been treated as an external charge.

\section{B. Compensating field}

The idea of Paper $\mathrm{I}^{3}$ was to eliminate the finite field in the solid slab by imposing a biasing field $\mathrm{E}_{\mathrm{CNC}}$. This field was found by requiring that the potential over the crystal slab vanishes. Similar to Eq. (12) for the potential over the entire periodic cell, the potential over the crystal can be interpreted in terms of the average electric field,

$$
E_{d}=-\frac{n-1}{2 n} E_{2}+\frac{n+1}{2 n} E_{1} .
$$

Setting $E_{d}=0$ gives a linear relation between $E_{1}$ and $E_{2}$, namely,

$$
(n-1) \mathrm{E}_{2}=(n+1) \mathrm{E}_{1} \text {. }
$$

At this point, we finally invoke the linear constitutive relations $4 \pi \mathrm{P}_{\mathrm{H}}=\left(\epsilon_{\mathrm{H}}-1\right) \mathrm{E}_{\mathrm{H}}$ for the polarization in the Stern layer and $4 \pi \mathrm{P}_{1}=\left(\epsilon_{\mathrm{d}}-1\right) \mathrm{E}_{1}, 4 \pi \mathrm{P}_{2}=\left(\epsilon_{\mathrm{d}}-1\right) \mathrm{E}_{2}$ for the polarization in the dielectric material between the crystal planes. Substituting in Eqs. (7)-(9) and combining with Eqs. (13)-(15), we have

$$
\begin{aligned}
\epsilon_{\mathrm{H}} E_{\mathrm{H}} & =4 \pi \sigma, \\
\epsilon_{\mathrm{H}} E_{\mathrm{H}}+\epsilon_{\mathrm{d}} E_{1} & =4 \pi \sigma_{0}, \\
\epsilon_{\mathrm{d}} E_{2}+\epsilon_{\mathrm{d}} E_{1} & =4 \pi \sigma_{0} .
\end{aligned}
$$

Equation (26) together with Eq. (23) gives

$$
\mathrm{CNC} \Rightarrow\left\{\begin{array}{l}
\mathrm{E} 2=\frac{4 \pi}{\epsilon \mathrm{d}}\left(\frac{n+1}{2 n}\right) \sigma 0(1) \\
\mathrm{E} 1=\frac{4 \pi}{\epsilon \mathrm{d}}\left(\frac{n 1}{2 n}\right) \sigma 0(2) .
\end{array}\right.
$$

Replacing $E_{1}$ in Eq. (25) with Eq. (28) and then substituting in Eq. (24) we recover Eq. (1). Inserting Eqs. (27) and (28) into Eq. (12), we find our expression for the compensating field,

$$
\mathrm{E}_{\mathrm{CNC}}=-\frac{4 \pi}{\epsilon_{\mathrm{H}}}\left(\frac{l_{\mathrm{H}}}{\mathrm{L}}\right)\left(\frac{n+1}{n}\right) \sigma_{0}
$$

Comparing Eq. (29) for the compensating field to Eq. (1) for $\sigma$ under CNC conditions $\left(E_{\mathrm{d}}=0\right)$, we note that all parameters of the continuum model except the number of planes $(n)$ have disappeared. This would suggest that the validity of Eq. (1) extends beyond the continuum model as was confirmed by the close agreement found for the FFMD model in Paper I. ${ }^{3}$
Equation (21) is generally valid whether under CNC conditions or not. When $\bar{E}=E_{\mathrm{CNC}}$, the induced charge is determined by the fixed charge $\sigma_{0}$ according to Eq. (1). Inserting this equation in Eq. (21) yields the value of the displacement field at $\mathrm{CNC}$,

$$
\mathrm{D}_{\mathrm{CNC}}^{\mathrm{ICS}}=-4 \pi\left(\frac{n+1}{2 n}\right) \sigma_{0},
$$

As hypothesised, the value of $D_{\mathrm{CNC}}$ is known a priori. The consequence of this is that $\mathrm{CNC}$ does not have to be located by scanning over E fields and performing an interpolation. This is crucial as in our previous work, this amounted to a minimum of 5 separate trajectories. Polarization in these systems approaches a value comparable to convergence after 100$200 \mathrm{ps}$, and while this is trivial for a classical code, our ambition is to include electronic structure, which will, in general, produce a significantly different value of $\mathrm{P}_{\mathrm{CNC}}$. This means the search over E fields would have to be repeated using DFTMD. By contrast, the model value of $D_{\mathrm{CNC}}$ will be the same in both FF and DFT descriptions.

\section{Equation of state}

In the EDL study of Ref. 8, we observed that the response of the polarization to a finite field was remarkably linear, even for relatively large fields. This suggested writing the electric equation of state in the linear form

$$
4 \pi \mathrm{P}^{\mathrm{ICS}}=4 \pi \gamma_{\mathrm{E}} \sigma_{0}+\left(\epsilon_{\perp}-1\right) \overline{\mathrm{E}}
$$

where $\gamma_{\mathrm{E}}$ and $\epsilon_{\perp}$ are constants. The interpretation of $\epsilon_{\perp}$ is as a "global" dielectric constant for the composite system. It was shown that the capacitance could be estimated without finding $\mathrm{CNC}$ by calculating the derivative of the potential with respect to the surface charge, opening a complimentary route to calculation of this important observable.

Here, the expression for the charge in the double layer is subjected to the same analysis. We first rewrite the expression for the Maxwell field [Eq. (12)] as the sum of the potential over the two Stern layers (assumed identical in our model) and the potential over the crystal [Eq. (22)],

$$
\overline{\mathrm{E}} L=-2 \mathrm{E}_{\mathrm{H}} l_{\mathrm{H}}+n \overline{\mathrm{E}}_{\mathrm{d}} R .
$$

With some manipulation of Eqs. (22) and (24)-(26), $E_{d}$ can be expressed as,

$$
\mathrm{E}_{\mathrm{d}}=-\frac{4 \pi}{\epsilon_{\mathrm{d}}}\left(\sigma-\frac{n+1}{2 n} \sigma_{0}\right)
$$

the difference between $\sigma$ and its value under CNC bias [Eq. (1)]. This is as expected because the average field in the crystal vanishes at CNC. Substituting in Eq. (32) gives $\sigma$ in the form $^{18}$

$$
\sigma=\left(\frac{n+1}{2} \frac{\sigma_{0}}{\mathrm{C}_{\mathrm{d}}}-\overline{\mathrm{E}} L\right)\left(\frac{2}{\mathrm{C}_{\mathrm{H}}}+\frac{n}{\mathrm{C}_{\mathrm{d}}}\right)^{-1},
$$

where $C_{d}=\epsilon_{d} /(4 \pi R)$ and $C_{H}=\epsilon_{H} /\left(4 \pi l_{H}\right)$. Substituting in Eq. (19) for the full polarization, we find the parameters of the equation of state in Eq. (31), 


$$
\begin{aligned}
& \epsilon_{\perp}=4 \pi L C_{\text {tot }}, \\
& \gamma_{\mathrm{E}}=-\left(\frac{n+1}{2}\right) \frac{C_{\text {tot }}}{C_{\mathrm{d}}} .
\end{aligned}
$$

$\mathrm{C}_{\text {tot }}$ is the series capacitance of polar slab including the two double layers at either end and defined as

$$
\frac{1}{\mathrm{C}_{\mathrm{tot}}}=\frac{2}{\mathrm{C}_{\mathrm{H}}}+\frac{n}{\mathrm{C}_{\mathrm{d}}} \text {. }
$$

Inspecting the large $n$ (thick slab) behavior of the equation of state reveals some surprising features. In this limit, the contribution $2 / \mathrm{C}_{\mathrm{H}}$ of the Helmholtz layer to series capacitance $\mathrm{C}_{\text {tot }}$ [Eq. (37)] can be neglected. This reduces the composite dielectric constant Eq. (35) to $\epsilon_{\perp}=(L / n R) \epsilon_{\mathrm{d}}$. This would imply that the value $\epsilon_{\perp}$ could decrease below unity, which is a forbidden interval for dielectric constants. However, $L=n R$ $+2 l_{\mathrm{H}}$ coupling box length and slab width. Rigorously, using the definitions of $\mathrm{C}_{\mathrm{H}}$ and $\mathrm{C}_{\mathrm{d}}$,

$$
\frac{1}{4 \pi C_{\text {tot }}}=\frac{2 l_{\mathrm{H}}}{\epsilon_{\mathrm{H}}}+\frac{n \mathrm{R}}{\epsilon_{\mathrm{d}}}<2 l_{\mathrm{H}}+n \mathrm{R}<\mathrm{L},
$$

which guarantees that $\epsilon_{\perp}>1$. To investigate the large $n$ limit of $\gamma_{E}$, we write Eq. (36) as

$$
\gamma_{\mathrm{E}} \sigma_{0}=-\frac{n \mathrm{C}_{\mathrm{tot}}}{\mathrm{C}_{\mathrm{d}}} \sigma_{\mathrm{CNC}},
$$

where we have made use of Eq. (1). For large $n$, the prefactor approaches unity leading to $\gamma_{\mathrm{E}} \sigma_{0}=-\sigma_{\mathrm{CNC}}$. This is rather surprising because Eq. (1) is derived by imposing CNC conditions, while the equation of state Eq. (32) is generally valid (given the linear response approximation of the continuum model). Evidently the zero field polarization $(\bar{E}=0)$ converges to the $\mathrm{CNC}$ value for increasing slab width.

The main objective of our study of the EDL in Ref. 8 was the computation of the capacitance $\mathrm{C}_{\mathrm{H}}$ of the Stern layer. This quantity is also defined for the polar surface as can be seen by rewriting Eq. (29) as

$$
\Delta \mathrm{V}_{\mathrm{CNC}}=-\mathrm{LE}_{\mathrm{CNC}}=\frac{2}{\mathrm{C}_{\mathrm{H}}} \sigma_{\mathrm{CNC}} .
$$

$\Delta \mathrm{V}_{\mathrm{CNC}}$ is the potential over the periodic cell. The potential difference over the crystal vanishes at $\mathrm{CNC}$. The potential over the electrolyte is always zero, and therefore $\Delta \mathrm{V}_{\mathrm{CNC}}$ is the sum of the potentials over the compact double layers. Recall, however, that for the polar surface, the double carries a net charge and Eq. (40) must be regarded as a formal definition of the double capacitance.

$\mathrm{C}_{\mathrm{H}}$ can be estimated directly from Eq. (40). As an alternative, we considered in Ref. 8 to estimate the compact layer capacitance from the response to the variation in $\bar{D}$ given by the conjugate form of Eq. (31),

$$
4 \pi \mathrm{P}^{\mathrm{ICS}}=4 \pi \gamma_{\mathrm{D}} \sigma_{0}+\left(1-\frac{1}{\epsilon_{\perp}}\right) \overline{\mathrm{D}}
$$

where $\gamma_{D}=\gamma_{E} / \epsilon_{\perp}$. This is significant because the aqueous response to the $\bar{D}$ field is known to converge faster than its conjugate by a factor equal to its dielectric constant. However, depending on the actual form of the estimator, the gain in the convergence of the polarization does not always lead to a speed-up of the calculation of dielectric properties and a good example is the direct application of constant D simulations to compute the dielectric constant of liquid water. ${ }^{12}$ We will come back to this issue in Sec. VI.

A third option for computing the double layer capacitance is from the value of polarization at $\mathrm{CNC}$. Writing the polarization at $\mathrm{CNC}$ as the difference between the corresponding values of the displacement field [Eq. (30)] and the Maxwell [Eq. (40)], we can find

$$
\mathrm{C}_{\mathrm{H}}=\frac{\sigma_{\mathrm{CNC}}}{2 \pi \mathrm{L}}\left(\sigma_{\mathrm{CNC}}+\mathrm{P}_{\mathrm{CNC}}^{\mathrm{ICS}}\right)^{-1} .
$$

The results for the capacitance of the double layer reported later were calculated using Eq. (42) although also suffering from deterioration in accuracy in the limit of large width. $\mathrm{P}_{\mathrm{CNC}}^{\mathrm{ICS}}$ approaches $-\sigma_{\mathrm{CNC}}$ as $1 / \mathrm{L}$, keeping $\mathrm{C}_{\mathrm{H}}$ finite, but with increasing statistical error.

\section{ELECTRONIC POLARIZATION}

The modern theory of polarization was developed by Vanderbilt, Resta, and colleagues to describe and compute electronic polarization in insulating solids (semiconductors, ionic crystals) in the framework of the Density Functional Theory (DFT) based band structure calculation in periodic supercells. $^{9-11}$ As had been realized already for some time (see, for example, the work of Martin ${ }^{17}$ ), polarization in such systems cannot be computed from the electronic density but must be treated as an independent system variable. The breakthrough came when this variable was identified by Vanderbilt and coworkers as a Berry phase related to the Bloch orbitals in reciprocal space. ${ }^{9}$ Resta subsequently reformulated the Berry phase for polarization in terms of a phase in real space. ${ }^{19,20}$ The implementation of Berry-phase electronic polarization in the $\mathrm{CP} 2 \mathrm{~K}$ code employed in the present calculation is based on the $\Gamma$-point-only Resta approach. ${ }^{21}$ An alternative real approach for the calculation of electronic polarization uses Maximally Localized Wannier Functions (MLWFs). ${ }^{22-24}$ The polarization is obtained from the dipole moment of the centers of the MLWFs. Polarization in this definition is again multivalued because of the freedom to choose which periodic image to use in common with the classical itinerant polarization of Sec. II B.

This poses a practical problem of branch alignment. The $n \Delta_{q} \mathrm{P}$ gauge of the polarization upon initialization of the electron structure calculation is not easy to control. However, as pointed out in Sec. II B, it is crucial that the gauge of the displacement field in the constant D Hamiltonian matches the gauge of the polarization. The challenge is now: how to map the Berry phase polarization being handled by the computer code to the value we recognize as being consistent with our model? For this, we consider our initial geometry in the basis of the Wannier-center representation of the electronic wavefunction. The localized electron pairs are attached to nearby 
atoms, and these atoms are wrapped into the box as whole molecules. This "molecular gauge" gives us the anchoring value of the polarization and differs from other polarization branches by $n \Delta_{q} P$ for integer $n$.

In principle, we could then go ahead with the ICS geometry. However, since solvent molecules are not defined beforehand-as in the case of FFMD simulation-and are free to straddle across the supercell boundary or (more seriously) dissociate, we would have to keep track of a frequently changing polarization branch. This can be avoided by conveniently shifting one-half box length to the ECS. Now, no aqueous species can cross the boundary, and since the crystal will be held fixed, neither will any part of the lattice. The only the change in the branch will be from the electrons within the crystal crossing the boundary to localize on a different plane of ions.

By performing this translation of the supercell from ICS to ECS, we have of course altered $P_{\text {cell }}$. Since the value of $D_{C N C}$ is derived for a given $P, D_{C N C}^{E C S}$ can indeed be different to $D_{C N C}^{I C S}$. What is more, the boundaries of the cell (Fig. 1) can either be located in an interplanar layer with electric field $E_{1}$ or in a layer with electric field $E_{2}$. We shall refer to these as ECS1 and ECS2, respectively. They have different $P_{\text {cell }}$ values and also different boundary contributions of the kind introduced in Eq. (18). We must therefore repeat the derivation of Sec. III A. This derivation is technical, but also proof that the method is consistent; the very interested reader can find it in the Appendix to the main body of text. It turns out that for ECS1, the value must be corrected by $\sigma_{0}$ and becomes

$$
\mathrm{D}_{\mathrm{CNC}}^{\mathrm{ECS} 1}=4 \pi \frac{n-1}{2 n} \sigma_{0} .
$$

In this study, we will use a DFTMD crystal slab of only $n=3$ and so $(n+1) / 2$ is even and we could draw the cell boundary in the central $E_{2}$ layer. It transpires that the displacement field D is the same for this ECS2 as for the ICS. So, in

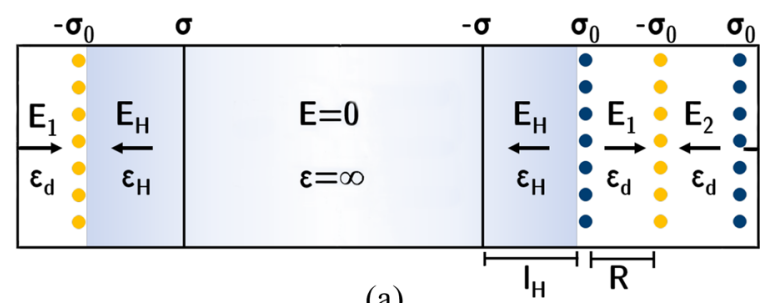

(a)

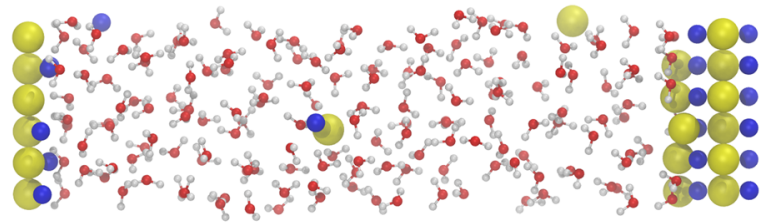

(b)

FIG. 2. Above: Stern model of the ECS. The lone plane on the left-hand side means that $E_{1}$ is cut by the supercell boundary and so this is an ECS1 setup. All the symbols have the same meaning as in Fig. 1. Below: MD snapshot of the $\mathrm{NaCl}(111) / \mathrm{NaCl}$ solution system, with $\mathrm{Na}^{+}$in blue and $\mathrm{Cl}^{-}$in yellow. order to demonstrate this supercell dependence, we shall shift by one plane to ECS1, as shown in Fig. 2. We shall therefore apply the smaller, positive D of Eq. (43).

\section{COMPUTATIONAL DETAILS}

From our previous work, we found that the model agreed remarkably well with the results from FFMD with as few as 4 planes of ions $(n=3)$. We therefore feel confident to use this size of system in the DFTMD. Furthermore, we reduced the lateral extent of the cell by reducing the number of ions per plane $\left[\mathrm{N}_{0}\right.$ in Eq. (6)] from 16 to 9 . This also allows us to reduce to 7 aqueous ion pairs (previously 20) and still retain an electrolyte region. This is because the compensating charge is $A \sigma_{0}(n+1) / 2 n=6 e$. We can now reduce the $z$ axis without significantly affecting the initial concentration. Note that since the hexagonal symmetry of the (111) plane requires one of the dimensions to be an even number of planes, this manoeuvre introduced a stacking fault along the $x$ axis. This leads to a system of 539 atoms within a box of lengths [1.197, 1.0365, $4.75] \mathrm{nm}$. We recomputed the capacitance for this smaller size to be $8.5 \mu \mathrm{Fcm}^{-2}$, a minor increase of $3 \%$ from the previous value of $8.2 \mu \mathrm{Fcm}^{-2}$ for the system without the stacking fault in Paper $\mathrm{I}^{3}$ We then tested doubling the lateral extent, and this yields $8.4 \mu \mathrm{Fcm}^{-2}$. This deviation is small enough to justify our choice of minimalistic supercell. All FFMD simulations were performed under ambient conditions using a modified version of the GROMACS 4 package. ${ }^{25}$ The water model was Simple Point Charge/Extended (SPC/E), ${ }^{26}$ with the $\mathrm{Na}^{+}$and $\mathrm{Cl}^{-}$parameters of Joung and Cheatham, ${ }^{27}$ which has been validated for high ionic strength. ${ }^{28,29}$ Technical settings of the simulations were the same as used in Paper I. ${ }^{3}$ In the analysis of the trajectories, the first 200 ps were discarded unless otherwise specified.

The DFTMD simulations were performed with CP2K. ${ }^{21,30}$ The pseudo-potentials used were Goedecker-Teter-Hutter $(\mathrm{GTH}){ }^{31}$ with the double-zeta polarized DZVP-MOLOPT-SRGTH basis set, ${ }^{32}$ such that the nuclei of $\mathrm{Na}, \mathrm{Cl}, \mathrm{O}$, and $\mathrm{H}$ have apparent charges of $9 e, 7 e, 6 e$, and $1 e$, respectively. The exchange correlation functional was Perdew-BurkeErnzerhof (PBE), ${ }^{33}$ the time step was $0.5 \mathrm{fs}$, the BussiParrinello thermostat was set to be $298 \mathrm{~K}$ with a time constant of $20 \mathrm{fs},{ }^{34}$ Orbital Transformation (OT) was used with full single inverse with default $(-1)$ step size and energy gap, and convergence was $5 \times 10^{-7} .35$ A charge cutoff of 320 Ry with 40 Ry for the relative grid was found to be sufficient. The constant D implementation in CP2K can be referenced to Ref. 36 and is publicly available.

\section{RESULTS AND DISCUSSION}

\section{A. FFMD validation}

The small system to be submitted to DFTMD was first studied with FFMD in order to verify the derived value of $\mathrm{D}_{\mathrm{CNC}}$ [Eq. (43)]. As in the previous work, a scan over E fields was performed, and an interpolation of the change in potential over the crystal was found $E_{\mathrm{CNC}}$ to be $-3.82 \mathrm{~V} \mathrm{~nm}^{-1}$, as shown in Fig. 3. 


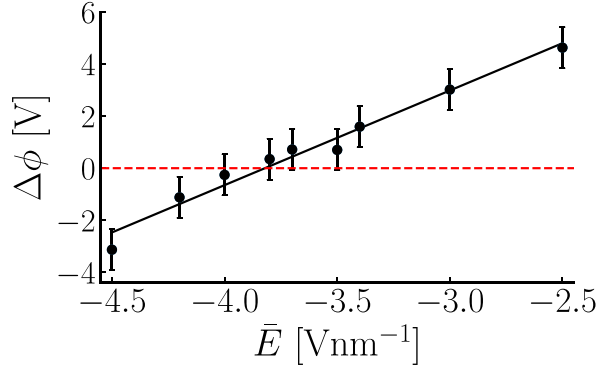

FIG. 3. FFMD simulations of the $n=3$ ECS1 of Fig. 2. $E_{\text {CNC }}$ was found to be -3.82 $\mathrm{Vnm}^{-1}$. Error bars are $3 \sigma$ obtained by jackknife resampling of the data.

For this system, $D_{\mathrm{CNC}}^{\mathrm{ECS1}}=43.75 \mathrm{~V} \mathrm{~nm}^{-1}=24.18 \times 10^{-3} e \AA^{-2}$. Therefore, we expected the polarization at CNC to be 26.34 $\times 10^{-3} e \AA^{-2}$. Calculating the polarization as simply the sum of the classical charges multiplied by their positions (per unit volume) gave the slightly larger $26.78 \times 10^{-3} e \AA^{-2}$ for the point at $3.8 \mathrm{~V} \mathrm{~nm}^{-1}$, showing that our theoretical value of $\mathrm{D}_{\mathrm{CNC}}$ was accurate. We then ran the same $\mathrm{CNC}$ simulation but at a constant displacement field. We found $\mathrm{P}_{\mathrm{CNC}}=26.26 \times 10^{-3} e \AA^{-2}$ with a corresponding $E_{\mathrm{CNC}}=-3.76 \mathrm{~V} \mathrm{~nm}^{-1}$. The comparison is shown in Fig. 4.

Therefore, the result of the constant D approach is consistent with that of the E field interpolation and the difference was of the order $0.05 \mathrm{~V} \mathrm{~nm}^{-1}$. This validates Eq. (43) and the associated Eq. (30). Furthermore, it can be clearly seen in Fig. 4 that the $D_{\mathrm{CNC}}$ field ensemble achieves a much faster convergence of the polarization over its thermodynamic conjugate condition $\mathrm{E}_{\mathrm{CNC}}$.

\section{B. DFTMD simulations}

\section{Initialization and polarization alignment}

We carried over the last frame of the FFMD trajectory to be the first frame of DFTMD simulations, after a short geometry optimization. Because of the multi-valued nature of polarization, the first task was to align the starting value of $P$

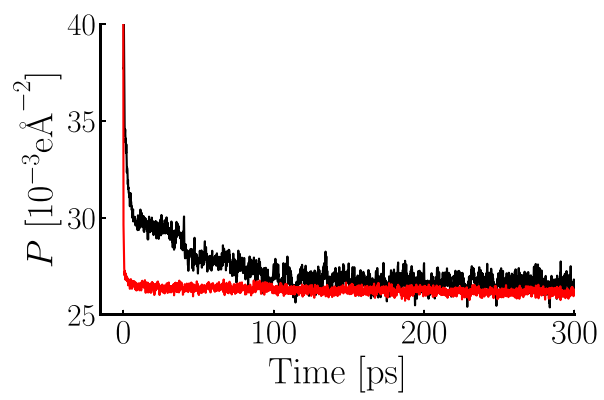

FIG. 4. FFMD simulations of the $n=3$ ECS1 of Fig. 2. The red line is $D=43.75 \mathrm{~V}$ $\mathrm{nm}^{-1}$, while the black line is $\bar{E}=-3.8 \mathrm{Vnm}^{-1}$. Only the first $300 \mathrm{ps}$ are displayed to emphasize the behavior in the range $0-100 \mathrm{ps}$, and the final values of $P$ are given in the text. calculated from the Berry phase formalism to the anchoring polarization calculated from the maximally localized Wannier functions (MLWFs) ${ }^{22,24}$ as explained in Sec. IV. The two values of polarization can differ by a multiple of the quantum of polarization $\Delta_{q} P$. Using Eq. (6), we computed a $\Delta_{q} \mathrm{P}=8.06$ $\times 10^{-3} e \AA^{-2}$ for our setup. A test calculation (red in Fig. 5) showed that $\mathrm{CP} 2 \mathrm{~K}$ had calculated the starting polarization as $1.42 \times 10^{-3} e \AA^{-2}$. By aligning this value to the molecular gauge obtained from MLWFs which is $25.61 \times 10^{-3} e \AA^{-2}$, we found out that the starting value of $P$ in our DFTMD system differs by $3 \Delta_{q} P$.

$\mathrm{D}_{\mathrm{CNC}}$ depends only on $\sigma_{0}$ and the number of crystal planes as indicated by Eq. (43). For $n=3, D_{\mathrm{CNC}}=4 \pi \sigma_{0} / 3$ $=12 \pi \Delta_{q} P$ where $\sigma_{0}=N_{0} \Delta_{q} P=9 \Delta_{q} P$ for our small system. This value is the same for both FFMD and DFTMD simulations in our setup. As pointed out already in Ref. 8, and reiterated in Sec. II $B$, the displacement field inherits the multivalued nature of $P$. This means the branch shift of $3 \Delta_{q} P$ as found by aligning the polarization needs to be accounted for when imposing the $D$ value in the constant $D$ simulation. Specifically, the actual branch-matched $\mathrm{D}_{\mathrm{CNC}}$ in our case differs from the theoretical target $D_{\mathrm{CNC}}$ by $4 \pi\left(3 \Delta_{q} \mathrm{P}\right)$, which is by coincidence equal to the theoretical $D_{\mathrm{CNC}}=12 \pi \Delta_{q} P$ itself. In other words, applying $D=0$ should restore the $\mathrm{CNC}$ state for our setup.

\section{Capacitance and dielectric response}

With above considerations in minds, DFTMD at D $=0$ was propagated for $\sim 10$ ps (Fig. 5), with the first 1 ps discarded as an additional equilibration time. The remaining 9 ps were used to calculate $\langle\mathrm{P}\rangle$. The polarization was found to be 24.86 $\times 10^{-3} e \AA^{-2}$. Using Eq. (42) and adjusting the branch shift of $3 \Delta_{q} \mathrm{P}$, one gets a capacitance of $26.38 \mu \mathrm{Fcm}^{-2}$. This is to be compared with the $8.66 \mu \mathrm{Fcm}^{-2}$ from FFMD with the polarization of $26.26 \times 10^{-3} e \AA^{-2}$.

A factor of three difference between capacitance values may seem counterintuitive because of only a relatively small $\sim 10 \%$ difference in the polarizations. However, this is indeed

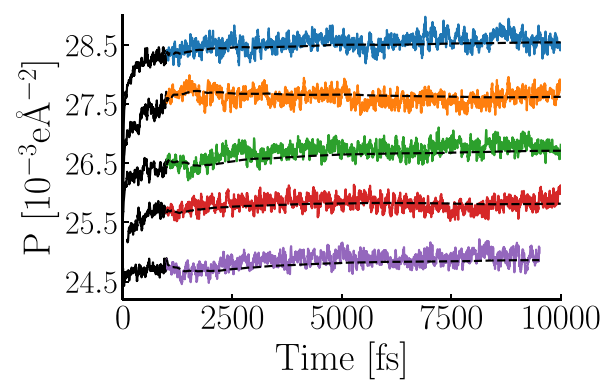

FIG. 5. Polarization time-series data from the DFTMD trajectories. The first 1 ps was discarded (black solid line). The cumulative average is shown as the black dashed line. The red (second to last) series had its very early fs behavior removed as it was very erratic, likely due to the SCF converging on a higher energy state. The blue (top) series crosses the Berry phase boundary during the trajectory and has been unwrapped. 


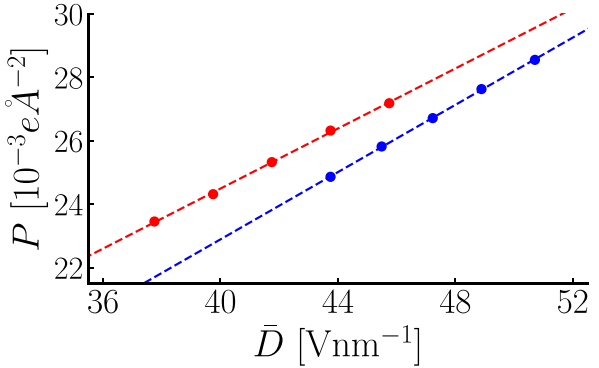

FIG. 6. Constant displacement field simulations. CNC is at $43.75 \mathrm{~V} \mathrm{~nm}^{-1}$. The red (top) series is FFMD, and the blue (bottom) series is DFTMD. The gradients are 0.47325 and 0.53010 , respectively.

the case and due to the form of Eq. (42) in which $\delta \mathrm{C}_{\mathrm{H}} / \mathrm{C}_{\mathrm{H}}$ is unfavorably scaled up by $\mathrm{C}_{\mathrm{H}}$. One might therefore be concerned about the convergence of $\mathrm{C}_{\mathrm{H}}$ calculated from DFTMD. To estimate this error, we randomly took 10 uncorrelated windows of 9 ps from FFMD and calculated a standard deviation of $0.044 \times 10^{-3} e \AA^{-2}$. This translates to $2 \%$ error in the calculated $\mathrm{C}_{\mathrm{H}}$ from FFMD and an estimation of $6 \%$ error in the calculated $\mathrm{C}_{\mathrm{H}}$ from DFTMD.

In order to compute $\epsilon_{\perp}$ which serves as the overall dielectric constant of the composite system, we also carried out four additional constant D DFTMD simulations at different $D$ values between zero and the Zener breakdown voltage (Fig. 5). These data are compared with those obtained from FFMD in Fig. 6. From Eq. (41), the gradient gave $\epsilon_{\perp}$ as 7.6 and 24.5 for FF and DFT systems, respectively. The ratio is roughly the same ratio as for the $\mathrm{C}_{\mathrm{H}}$ estimates, which is just a coincidence. According to Eq. (35), $\epsilon_{\perp}$ is determined by the leading term $C_{d}$ since the ionic solid and EDLs can be viewed as capacitors connected in series and $n$ is always larger than 2 . This is also the reason why the finite size effect which plagues the computation of $\mathrm{C}_{\mathrm{H}}$ is so serious. The charge planes in solid $\mathrm{NaCl}$ in FFMD simulation are separated by vacuum $\left(\epsilon_{\mathrm{d}}=1\right)$. However, DFTMD simulation includes electronic polarization. The optical dielectric constant of $\mathrm{NaCl}$ solid with this functional has been reported to be 2.49. ${ }^{37}$ Thus, it is the electronic polarization which causes the factor 3 difference in $\epsilon_{\perp}$.
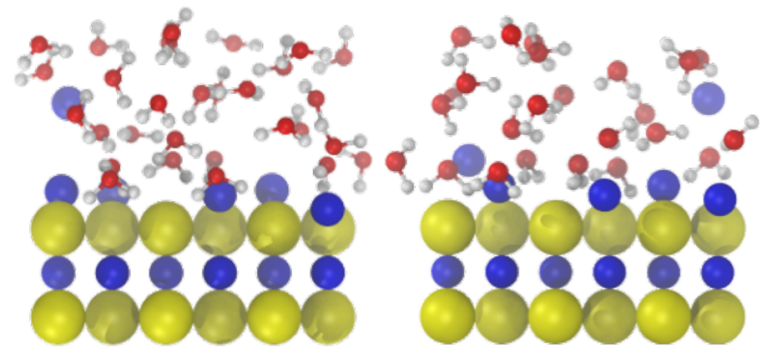

FIG. 7. MD snapshots from FFMD (left) and DFTMD (right) simulations of the $\mathrm{NaCl}(111) / \mathrm{NaCl}$ solution system at $D_{\mathrm{CNC}}$. The FFMD snapshot is the starting geometry for the DFTMD. The snapshot on the right can be seen to have a greater sodium-surface separation; see Fig. 8 for quantitative detail.
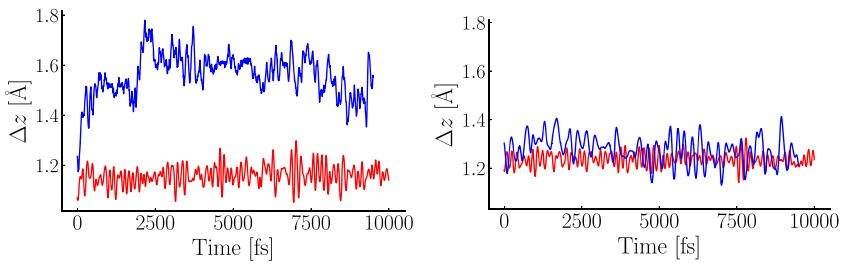

FIG. 8. Constant displacement field simulations. Right: aqueous sodium; left: aqueous chloride. The red (bottom) series are FFMD, and the blue (top) series are DFTMD. The z-positions of the 5 inner-shell ions have been averaged and are displayed relative to the fixed surface plane. Both FFMD and DFTMD simulations have the same starting geometry other than a short geometry optimization for initiating DFTMD.

In Paper $\mathrm{I}^{3}$ the difference in capacitance between the polar $\mathrm{NaCl}(111)$ and non-polar $\mathrm{NaCl}(100)$ was rationalized in terms of the double layer structure. Here again we see a plane of inner-sphere counter-ions on both sodium and chlorine sides of the $\mathrm{NaCl}(111)$ surface as the main component of the EDLs (Fig. 7). Distances between the inner-sphere counterions and the polar surfaces are plotted in Fig. 8. As one can see, the distance between $\mathrm{Cl}^{-}$(solution) and $\mathrm{Na}^{+}$(surface) and the distance between $\mathrm{Na}^{+}$(solution) and $\mathrm{Cl}^{-}$(surface) are actually smaller than the layer-wise distance in the ionic crystal in the [111] direction which is $1.63 \AA$. For the case of FFMD simulations, the distances between the inner-sphere counter-ions and $\mathrm{NaCl}(111)$ surfaces are about $1.2 \AA$. Such a distance in vacuum will lead to a theoretical capacitance of $7.4 \mu \mathrm{Fcm}^{-2}$ which is rather close to the one calculated from the simulation $\left(8.7 \mu \mathrm{Fcm}^{-2}\right)$. For the case of DFTMD simulation, the distance for $\mathrm{Cl}^{-}$(solution) and $\mathrm{Na}^{+}$(surface) is quite similar to that from FFMD simulations, but the distance between $\mathrm{Na}^{+}$ (solution) is about 50\% larger than that from FFMD simulations. Therefore, the capacitance of polar $\mathrm{NaCl}(111)$ calculated from DFTMD simulations should be smaller when compared to that from FFMD if it solely depended on the ionic structure. Instead, the opposite was found, and $C_{H}$ from DFTMD vs. FFMD is $26.4 \mu \mathrm{Fcm}^{-2}$ vs. $8.7 \mu \mathrm{Fcm}^{-2}$. Although there is indeed a contribution to $\mathrm{C}_{\mathrm{H}}$ from outer-sphere counter-ions (Fig. 7), the inner-sphere contribution will be the dominant part in direct analogy to the dead-layer effect of nanoconfined water. $^{38}$ This suggests that it is the electronic polarization present in the DFTMD simulations which determines the final value of $\mathrm{C}_{\mathrm{H}}$ at the $\mathrm{NaCl}(111) / \mathrm{NaCl}$ solution interface.

\section{CONCLUSIONS}

Stabilization mechanisms for type III polar surfaces depend on the physical conditions which the surfaces are exposed to. In the case of solid-vacuum and solid-solid interfaces, non-stoichiometric reconstruction and electronic reconstruction are the options. When in contact with electrolytes, such surfaces can be stabilized by supplying the compensating charge in the form of counter-ions from solution, preserving the composition of the solid surface. This is the stabilization mechanism we have investigated here using the $\mathrm{NaCl}(111)$ /aqueous $\mathrm{NaCl}$ solution as a prototype system. 
The challenges to study such polar ionic solid/electrolyte systems are two-fold: the inevitable finite size errors of atomistic models and the time scale needed to converge the calculation, particularly in the case of DFTMD simulations. We overcame the first challenge in the previous work by imposing a compensating electric field to locate the CNC state and validated the method with FFMD simulations. ${ }^{3}$

In this work, we expanded our study to DFTMD simulations of the same system and tackled the second challenge with constant electric displacement $\mathrm{D}$ simulations. The theoretical formula of $\mathrm{D}_{\mathrm{CNC}}$ which involves only structural parameters [Eq. (43) and associated equations] was first validated against FFMD simulations and then transferred to DFTMD simulations. Despite the fact that the estimator of $\mathrm{C}_{\mathrm{H}}$ of polar surfaces at $D_{\mathrm{CNC}}$ suffers from unfavorable error propagation, it is feasible to obtain results of a reasonable accuracy within the commonly accessible time scale of DFTMD (tens of picoseconds).

Comparing results of the Helmholtz capacitance $C_{H}$ between FFMD and DFTMD simulations for the same supercell of $\mathrm{NaCl}(111) / \mathrm{NaCl}$ solution system, it is found that $\mathrm{C}_{\mathrm{H}}$ is dominated by inner-sphere counter-ion contributions with the electronic polarization of the ionic solid determining the resulting value. This suggests that DFTMD is indispensable in modeling the charge compensation phenomena and EDLs at polar ionic solid/electrolyte interfaces. This needs to be backed up by a detailed structural analysis which has not been attempted in the present calculation focused on methodology and dielectric response. Because of its relevance to precipitation and nucleation, this work further hints that the electronic polarization needs to be taken into account when investigating the thermodynamics and kinetics of these processes. A related field where our finite field methods might be of use is that of nano-electrochemistry and nano-ionics. ${ }^{39-41}$

\section{ACKNOWLEDGMENTS}

The authors thank Jürg Hutter (University of Zurich) for getting the $\mathrm{CP} 2 \mathrm{~K}$ code ready for this application. T.S. is supported by a departmental studentship (No. RG84040) sponsored by the Engineering and Sciences Research Council (EPSRC) of the United Kingdom. Computational resources were provided by the UK Car-Parrinello (UKCP) consortium funded by EPSRC. This project was the subject of a HPC-Europa3 grant to T.S., and we thank the local support from KTH-PDC (Sweden). C.Z. gratefully acknowledges Uppsala University for the support of a start-up grant and Åforsk foundation for a research Grant (No. 18-460). Funding from the Swedish Strategic e-Science program eSSENCE is also acknowledged.

\section{APPENDIX: ELECTROLYTE CENTERED CELL DERIVATION}

Recall Fig. 1. We start with ECS1 which is the easier to understand. To begin, one cell boundary is placed just below the surface plane in the first $E_{1}$ layer. With one side of the supercell contained only one crystal plane, the other side must contain the remaining $n$ ion planes. The polarization of this cell (total dipole moment divided by boxlength $\mathrm{L}$ ) is now computed as

$$
\mathrm{P}_{\text {cell }}^{\mathrm{ECS} 1}=\frac{1}{\mathrm{~L}}\left(-\sigma_{\mathrm{H}} l_{e}+\sigma_{1}\left(2 l_{\mathrm{H}}+l_{e}\right)+\sigma_{2} \frac{n-1}{2} \mathrm{R}\right),
$$

where we have augmented the supercell superindex to indicate the type of cut. $l_{e}$ is the length of the electrolyte region, and therefore

$$
\mathrm{L}=l_{e}+2 l_{\mathrm{H}}+n R \text {. }
$$

The interface equations for the net charges are independent of cell geometry, so we can still use Eqs. (13)-(15). Substituting gives

$$
4 \pi P_{\text {cell }}^{\mathrm{ECS} 1}=\frac{1}{\mathrm{~L}}\left(\mathrm{E}_{\mathrm{H}} 2 l_{\mathrm{H}}+\mathrm{E}_{1} \mathrm{~L}-\mathrm{E}_{1} n \mathrm{R}+\left(\mathrm{E}_{1}+\mathrm{E}_{2}\right) \frac{n-1}{2} \mathrm{R}\right),
$$

where we have eliminated $l_{e}$ using the geometric relation Eq. (A2). The Maxwell field $\bar{E}$ consists of a sum of potentials and is not affected either by a change in cell boundaries ( $\bar{E}$ must be the same because it acts as a force on the particles and is therefore an observable). Thus using Eq. (12), we can write

$$
4 \pi P_{\text {cell }}^{\mathrm{ECS} 1}=\mathrm{E}_{1}-\overline{\mathrm{E}},
$$

which has an additional term of $E_{1}$ on the right-hand side when compared to the ICS version of Eq. (17).

As with the ICS, the ECS polarization is only complete after adding the appropriate cell boundary surface term. The field in the dielectric intersected by the boundary is $E_{1}$, and the surface term is therefore $P_{1}$. Adding to Eq. (A4) and combining with the $E_{1}$ term using constitutive relation yield

$$
4 \pi \mathrm{P}^{\mathrm{ECS} 1}=\epsilon_{\mathrm{d}} \mathrm{E}_{1}-\overline{\mathrm{E}} .
$$

This expression was derived for a specific choice of $E_{1}$ cut. One of the two sections of the crystal in the cell consists only of one ionic plane, a surface plane. A more evenly dividing $E_{1}$ cut can be generated by moving over pairs of neighbour planes leaving the outer planes in place. Such a pair of planes is charge neutral. Translating the pair will not alter the total dipole moment, and Eq. (A5) remains valid.

To find the expression for the polarization of an $E_{2}$ cut, we start again placing one cell boundary as near as possible to a surface plane. For an $E_{2}$ cut that is the second subsurface layer between the second and third plane of ions, the total dipole moment Eq. (A1) is modified to

$$
\begin{aligned}
\mathrm{P}_{\text {cell }}^{\mathrm{ECS} 2}= & \frac{1}{\mathrm{~L}}\left(-\sigma_{\mathrm{H}} l_{e}+\sigma_{1}\left(2 l_{\mathrm{H}}+l_{e}\right)\right. \\
& \left.-\sigma_{2}\left(2 \mathrm{R}+2 l_{\mathrm{H}}+l_{e}\right)-\sigma_{2} \frac{n-3}{2} \mathrm{R}\right) \\
= & \frac{1}{\mathrm{~L}}\left(-\sigma_{\mathrm{H}} l_{e}+\sigma_{1}\left(2 l_{\mathrm{H}}+l_{e}\right)-\sigma_{2} \mathrm{~L}+\sigma_{2} \frac{n-1}{2} \mathrm{R}\right) .
\end{aligned}
$$


Note that the direction of the dipole of a pair of the planes beyond the first two planes in Eq. (A6) is pointing in the opposite direction relative to the dipole of paired planes in Eq. (A1). Comparing to Eq. (A1), we see there is now an extra contribution $-\sigma_{2}$,

$$
\mathrm{P}_{\text {cell }}^{\mathrm{ECS} 2}=\mathrm{P}_{\text {cell }}^{\mathrm{ECS} 1}-\sigma_{2} .
$$

The cell polarization of the $E_{2}$ cut can therefore be immediately obtained from Eq. (A4) using again Eq. (15),

$$
4 \pi P_{\text {cell }}^{\mathrm{ECS} 2}=-\mathrm{E}_{2}-\overline{\mathrm{E}} .
$$

Perhaps not very surprising, the cell specific offset of $\bar{E}$ has changed from the field in the layer intersected by the $E_{1}$ cut to the corresponding field for an $E_{2}$ cut (note that the opposite sign is a result of the convention of the field directions in Fig. 1). The same applies to the surface term which is now given by $-\mathrm{P}_{2}$ leading in total to a cell polarization of

$$
4 \pi \mathrm{P}^{\mathrm{ECS} 2}=-\epsilon_{\mathrm{d}} \mathrm{E}_{2}-\overline{\mathrm{E}} \text {. }
$$

Again, paired planes can be moved to the other side. Equation (A9) is therefore the general expression for an $E_{2}$ cut. Subtracting the $E_{1}$ polarization Eq. (A5), we find

$$
\mathrm{P}^{\mathrm{ECS} 2}-\mathrm{P}^{\mathrm{ECS} 1}=-\frac{\epsilon_{\mathrm{d}}}{4 \pi}\left(\mathrm{E}_{1}+\mathrm{E}_{2}\right)=-\sigma_{0},
$$

where in the second step, we have substituted Eq. (26). This is to be expected that we have moved effectively one plane more to generate an $\mathrm{E}_{2}$ cut.

How to relate the ECS to ICS polarization? The $E_{2}$ cut turns out to lead the same polarization as for an ICS geometry. This can be seen by subtracting Eq. (26) from Eq. (25), eliminating $E_{1}$ and giving $\epsilon_{\mathrm{d}} E_{2}=\epsilon_{\mathrm{H}} E_{\mathrm{H}}$ which then via Eq. (24) becomes $\epsilon_{\mathrm{d}} \mathrm{E}_{2}=4 \pi \sigma$. Inserting in Eq. (A9), we obtain

$$
4 \pi \mathrm{P}^{\mathrm{ECS} 2}=-4 \pi \sigma-\overline{\mathrm{E}} .
$$

We indeed recover the same polarization as the ICS Eq. (19). This can be rationalized by counting the number of planes crossing the cell boundary when the supercell is shifted from the ICS to an ECS geometry. Every time a crystal plane leaves the cell and renters on the other side, the cell polarization jumps by $\pm \sigma_{2}$. The surface term alternates in step between its two values. For the $E_{2}$ variety of ECS cell, the numbers of jumps is even canceling each other. For the $E_{1}$ cut, the number of planes crossing the boundary is odd. The effective plane charge and boundary charge do not cancel but add to a net charge of $\pm \sigma_{0}$ depending on whether the shift is to the left or right.

The important conclusion of this exercise is that the displacement field values imposing CNC conditions, while derived from a continuum model, are in the end independent of the dielectric parameters $\epsilon_{\mathrm{d}}, \epsilon_{\mathrm{H}}$ and even of the geometric parameters $R, l_{H}$. The only parameter that matters is the bare charge density of the ionic planes. This gives us confidence that the relations for $\mathrm{D}$ are generic and can be transferred to our atomistic models. At present, however, we are not able to give a convincing "model free" explanation for this observation.

\section{REFERENCES}

${ }^{1}$ P. W. Tasker, J. Phys. C: Solid State Phys. 12, 4977 (1979).

2J. Goniakowski, F. Finocchi, and C. Noguera, Rep. Prog. Phys. 71, 016501 (2008).

${ }^{3}$ T. Sayer, C. Zhang, and M. Sprik, J. Chem. Phys. 147, 104702 (2017).

${ }^{4}$ C. Noguera and J. Goniakowski, Chem. Rev. 113, 4073 (2013).

${ }^{5}$ M. Stengel, N. A. Spaldin, and D. Vanderbilt, Nat. Phys. 5, 304 (2009).

${ }^{6}$ M. Stengel, D. Vanderbilt, and N. A. Spaldin, Phys. Rev. B 80, 224110 (2009).

${ }^{7}$ M. Sprik, Mol. Phys. 116, 3114 (2018).

${ }^{8}$ C. Zhang and M. Sprik, Phys. Rev. B 94, 245309 (2016).

${ }^{9}$ R. D. King-Smith and D. Vanderbilt, Phys. Rev. B 47, 1651 (1993).

${ }^{10}$ R. Resta, Rev. Mod. Phys. 66, 899 (1994).

${ }^{11}$ R. Resta and D. Vanderbilt, in Physics of Ferroelectrics: A Modern Perspective, Topics in Applied Physics, edited by K. M. Rabe, C. H. Ahn, and J.-M. Triscone (Springer-Verlag, 2007), Vol. 105, pp. 31-67.

${ }^{12}$ C. Zhang and M. Sprik, Phys. Rev. B 93, 144201 (2016).

${ }^{13}$ J.-M. Caillol, J. Chem. Phys. 101, 6080 (1994).

${ }^{14}$ S. W. de Leeuw, J. W. Perram, and E. R. Smith, Proc. R. Soc. A 373, 27 (1980).

${ }^{15}$ J.-M. Caillol, D. Levesque, and J. J. Weis, J. Chem. Phys. 91, 5544 (1989).

${ }^{16}$ J.-M. Caillol, D. Levesque, and J. J. Weis, J. Chem. Phys. 91, 5555 (1989).

${ }^{17}$ R. M. Martin, Phys. Rev. B 9, 1998 (1974).

${ }^{18}$ This is Eq. (6) of Paper $\mathrm{I},{ }^{3}$ which contained a typo.

${ }^{19}$ R. Resta, Phys. Rev. Lett. 80, 1800 (1998).

${ }^{20}$ R. Resta, J. Phys.: Condens. Matter 12, R107 (2000).

${ }^{21}$ J. Hutter, M. Iannuzzi, F. Schiffmann, and J. VandeVondele, Wiley Interdiscip. Rev.: Comput. Mol, Sci. 4, 15 (2014).

${ }^{22}$ N. Marzari and D. Vanderbilt, Phys. Rev. B 56, 12847 (1997).

${ }^{23}$ P. L. Silvestrelli, N. Marzari, D. Vanderbilt, and M. Parrinello, Solid State Commun. 107, 7 (1998).

${ }^{24}$ N. Marzari, A. A. Mostofi, J. R. Yates, I. Souza, and D. Vanderbilt, Rev. Mod. Phys. 84, 1419 (2012).

${ }^{25}$ B. Hess, C. Kutzner, D. van der Spoel, and E. Lindahl, J. Chem. Theory Comput. 4, 435 (2008).

${ }^{26}$ H. J. C. Berendsen, J. R. Grigera, and T. P. Straatsma, J. Phys. Chem. 91, 6269 (1987).

27. S. Joung and I. T. E. Cheatham, J. Phys. Chem. B 112, 9020 (2008).

${ }^{28}$ C. Zhang, S. Raugei, B. Eisenberg, and P. Carloni, J. Chem. Theory Comput. 6, 2167 (2010).

${ }^{29}$ I. Nezbeda, F. Mouka, and W. R. Smith, Mol. Phys. 114, 1665 (2016).

${ }^{30} \mathrm{~J}$. VandeVondele, M. Krack, F. Mohamed, M. Parrinello, T. Chassaing, and J. Hutter, Comput. Phys. Commun. 167, 103 (2005).

${ }^{31}$ S. Goedecker, M. Teter, and J. Hutter, Phys. Rev.B 54, 1703 (1996).

32J. VandeVondele and J. Hutter, J. Chem. Phys. 127, 114105 (2007).

${ }^{33}$ J. P. Perdew, K. Burke, and M. Ernzerhof, Phys. Rev. Lett. 77, 3865 (1996).

${ }^{34}$ G. Bussi, D. Donadio, and M. Parrinello, J. Chem. Phys. 126, 014101 (2007).

${ }^{35}$ J. VandeVondele and J. Hutter, J. Chem. Phys. 118, 4365 (2003).

${ }^{36}$ C. Zhang, J. Hutter, and M. Sprik, J. Phys. Chem. Lett. 7, 2696 (2016).

37J. H. Skone, M. Govoni, and G. Galli, Phys. Rev. B 89, 195112 (2014).

${ }^{38}$ C. Zhang, J. Chem. Phys. 148, 156101 (2018).

${ }^{39}$ I. Valov and W. D. Lu, Nanoscale 8, 13828 (2016).

${ }^{40}$ K. Terabe, T. Tsuchiya, R. Yang, and M. Aono, Nanoscale 8, 13873 (2016).

${ }^{41}$ S. M. Yang, A. N. Morozovska, R. Kumar, E. A. Eliseev, Y. Cao, L. Mazet, N. Balke, S. Jesse, R. K. Vasudevan, C. Dubourdieu, and S. V. Kalinin, Nat. Phys. 13, 812 (2017). 